\title{
Electricity market equilibrium models: The effect of parametrization
}

\author{
Ross Baldick \\ Department of Electrical and Computer Engineering \\ The University of Texas at Austin
}

\begin{abstract}
In this paper, I use an example from the literature to compare Cournot and supply function equilibrium models of bid-based electricity markets both with and without transmission constraints. I will demonstrate that the parametrization of the supply function model has a significant effect on the calculated results. In particular, several results reported in the literature are artifacts of assumptions in the parametrization of the model.

Keywords: Market power, Cournot equilibrium, supply function equilibrium, transmission constraints.
\end{abstract}

\section{INTRODUCTION}

In recent papers discussing electricity markets [1], [2], $[3],[4],[5],[6],[7]$ the authors present "supply function equilibrium" models of bidder interaction. This approach, pioneered by Green and Newbery [1], takes the development of supply function equilibrium by Klemperer and Meyer [8] and applies it to a bid-based pool (BBP) model. The BBP model is representative of energy market structure in a number of restructured electricity markets, such as England and Wales (until 2001), New Zealand, Australia, the (now defunct) Power Exchange in California, and the Pennsylvania-New Jersey-Maryland (PJM) interconnection.

The supply function equilibrium (SFE) models presented in [1], [2], [3], [4], [5], [6], [7] contrast with Cournot models of the electricity system [9], [10], [11], [12] in that SFE models of interaction better match what is explicitly required in the bid formats of typical BBP markets, which typically require the bidding of a supply function or a cost function. That is, the natural definition of economic equilibrium in a BBP market is the SFE condition that no player wants to unilaterally change its bid supply function.

Nevertheless, this literal connection between SFE models and BBP models does not necessarily imply that an SFE model is the best way to predict the likely outcome of strategic interaction between players. In the case that there are multiple SFEs, for example, the calculation of one SFE (of perhaps many) does not provide a convincing prediction of market outcome.

In the case of a BBP model applying to a single pricingperiod with no uncertainty of demand, there are almost inevitably multiple SFEs when players:

- have full discretion in choosing the parameters specifying their bid supply functions and

- are not constrained to bid the same supply function over multiple pricing-periods.

This observation is well-known in the economics literature and is explicit in the work of Klemperer and Meyer [8]; however, the implications do not seem to have been understood in the engineering literature. This paper attempts to clarify this issue in the context of a BBP.

In modeling the England and Wales BBP, Green and Newbery [1] modeled each player as specifying a single supply function bid that applied to all pricing-periods over an extended length of time. This requirement was true on a daily basis in the England and Wales pool until 2001.

However, in several BBP markets that were developed subsequently to the England and Wales market, such as the (now defunct) California PX, a different supply function can be specified for each half-hour or hour long pricingperiod. That is, the assumption in [1] of a single supply function applying across multiple pricing-periods is not a feature of all BBP markets. Assuming consistency of bid supply functions across multiple periods severely restricts the flexibility of bids compared to the true flexibility in these markets.

Furthermore, many of the results in the SFE literature involve restrictive parametrization of the bid cost functions. I will demonstrate with an example that the parametrization is critical to the results. In particular, with unrestricted parametrizations of the convex quadratic bid cost function, there are multiple equilibria.

The example serves to illustrate Klemperer and Meyer's general observation about multiple equilibria in the context of a BBP. In this sense, the result is not unexpected. However, there is a further and somewhat surprising effect of parametrization: a transmission constrained example will show that the existence or not of pure strategy equilibria can depend on assumptions about the parametrization of the bid cost functions.

It has been observed [13] that actual bids in the England and Wales pool resulted in price levels below that implied by the SFE model used by Green and Newbery and even further below that implied by Cournot models. This is an extremely important issue, but beyond the scope of this paper. See [14], [15] for a discussion of this issue.

The organization of the paper is as follows. Notation and the model are developed in the next section, with a detailed literature survey in section III. In section IV, I extend the transmission unconstrained version of an example by Weber and Overbye [4]. The transmission constrained version of the example system is then considered in section V. I conclude in section VI. 


\section{NotATION AND MODEL}

To fix some notation, consider a player $k$ that controls generation or demand at a bus. The net injection into the system by generation or demand under the control of player $k$ is denoted by $x_{k} \in \mathbb{R}$, where $\mathbb{R}$ is the set of real numbers.

I assume that for player $k$ the true costs of generation (or the negative of its true benefits in the case of a demander) are given by a quadratic function $\hat{f}_{k}: \mathbb{R} \rightarrow \mathbb{R}$ of the form:

$$
\forall x_{k} \in \mathbb{R}, \hat{f}_{k}\left(x_{k}\right)=\frac{1}{2} x_{k} \hat{R}_{k} x_{k}+\hat{c}_{k} x_{k},
$$

where $\hat{R}_{k} \in \mathbb{R}$ and $\hat{c}_{k} \in \mathbb{R}$ are, respectively, the coefficients of the quadratic and linear terms in the true cost function. That is, a caret over a function or parameter means the true value of that function or parameter.

I will also assume that the pool bidding rules require the bidding of a convex quadratic bid cost function $f_{k}: \mathbb{R} \rightarrow \mathbb{R}$ of the form:

$$
\forall x_{k} \in \mathbb{R}, f_{k}\left(x_{k}\right)=\frac{1}{2} x_{k} R_{k} x_{k}+c_{k} x_{k},
$$

where $R_{k} \in \mathbb{R}_{+}$is non-negative and $c_{k} \in \mathbb{R}$. The values of $R_{k}$ and $c_{k}$ specify the parametrization of the bid cost function. The condition that $R_{k}$ be non-negative enforces convexity of $f_{k}$. The pool performs economic dispatch or transmission constrained economic dispatch based on the bid cost functions. In terms of the SFE literature [8], the corresponding supply function $S_{k}: \mathbb{R} \rightarrow \mathbb{R}$ is defined by:

$$
\forall p_{k} \in \mathbb{R}, S_{k}\left(p_{k}\right)=\left[R_{k}\right]^{-1} p_{k}-c_{k} .
$$

I will develop the analysis in terms of bid cost functions since this fits most naturally with the BBP definition; however, I will refer to the resulting equilibrium as an SFE.

Typical BBP markets actually require either:

- a convex piecewise linear bid cost function (or equivalently, a piecewise constant, non-decreasing supply function) or

- a convex piecewise quadratic bid cost function (or equivalently, a piecewise linear, non-decreasing supply function.) In the former case, the convex quadratic function (1) can be interpreted as a smooth approximation to the piecewise linear function. Using a smooth approximation facilitates the application of first order optimality conditions to the pool optimization problem without resorting to considering the multiple segments in the bid. In the latter case that the BBP market requires a piecewise quadratic bid cost function, then the quadratic function (1) can be interpreted as matching a quadratic piece of the bid cost curve. As discussed by von der Fehr and Harbord, the assumption of a differentiable bid cost function may not be innocuous [16].

There are other possible ways to parameterize a bid cost function that are considerably more general than the convex quadratic function presented here. I will assume that a convex quadratic bid cost function is specified in the rules of the BBP market or that a convex quadratic function is a reasonable approximation to the possible bid functions.

In recent papers on SFE, the choice by player $k$ of the parameters $R_{k}$ or $c_{k}$ is often further restricted in some way in addition to requiring that $R_{k}$ be non-negative. The parameters that can be adjusted at will by the "strategic players" in a particular model are called the "strategic variables." To distinguish the previous literature in SFE models, I will describe four alternative specifications of the strategic variables, corresponding to different restrictions on the choices of the bid cost functions:

1. $c$-parametrization, where player $k$ can choose the linear coefficient $c_{k}$ in (1) arbitrarily but is required to specify a fixed, pre-chosen value of $R_{k}$. Sometimes, the fixed value of the quadratic coefficient $R_{k}$ is further assumed to be equal to the true value $\hat{R}_{k}$; however, I will also explore at length the case where the value of $R_{k}$ differs from $\hat{R}_{k}$.

2. $R$-parametrization, where player $k$ can choose $R_{k}$ to be an arbitrary non-negative value, but is required to specify a fixed, pre-chosen value of $c_{k}$. Sometimes the fixed value of $c_{k}$ is set to be zero.

3. $(R \propto c)$-parametrization, where player $k$ can choose $R_{k}$ and $c_{k}$ subject to the condition that $R_{k}$ and $c_{k}$ have a fixed linear relationship. That is, there is a fixed pre-chosen $\alpha_{k} \in \mathbb{R}$ and player $k$ must then choose $R_{k}$ and $c_{k}$ such that $R_{k}=\alpha_{k} c_{k}$. Typically, the linear relationship is the same as that between the true parameters $\hat{R}_{k}$ and $\hat{c}_{k}$, so that $\alpha_{k}=\hat{R}_{k} / \hat{c}_{k}$. In this case, the parametrization can be interpreted as allowing player $k$ to multiply its true cost function by an arbitrary non-negative constant.

4. $(R, c)$-parametrization, where player $k$ can choose both $R_{k}$ and $c_{k}$ arbitrarily.

Given the assumption that the BBP market requires convex quadratic bid functions (or that this is a reasonable approximation to the required bid format) then only the $(R, c)$ parametrization with $R_{k}$ non-negative represents the true flexibility available to bidders. The first three parametrizations restrict bidding compared to the true flexibility. As I will demonstrate, results from such restricted models may not be very good predictors of behavior under $(R, c)$ parametrization. In particular, the results from $c^{-}, R-$, and $(R \propto c)$-parametrization can be artifacts of the assumed values of the fixed parameters. In markets where bids can be even more general than allowed by $(R, c)$-parametrization, the results of $c$-, $R$-, and $(R \propto c)$-parametrization are likely to be even less predictive of the true range of equilibrium outcomes.

\section{LiterATURE SURVEY}

In this section, I review the literature in the context of the notation and model from section II. Several issues are raised, particularly regarding parametrization, that are investigated more fully in the latter sections.

Green and Newbery [1] and Green [2] assume that the linear cost coefficient $\hat{c}_{k}$ in the true cost function of every player is zero (or that the coefficients are all equal to same non-zero constant.) Green and Newbery adopt an $R$ parametrization model and concentrate on the case where $c_{k}=0$ and, as discussed above, assume that suppliers must bid the same function across multiple pricing-periods. This assumption was realistic on a daily basis for the England and Wales pool until 2001. 
Rudkevich [5] also assumes that the linear cost coefficient $\hat{c}_{k}$ in the true cost function of every player is zero. He also posits that each player $k$ must bid a single function that applies in all pricing-periods during a day. He then uses the observed supply response of the rest of the players over the day to construct an approximation to the bid supply function of the rest of the market. He then finds an optimal $(R, c)$-parametrization response for player $k$ to the supply function of the rest of the market, which turns out to have linear coefficient $c_{k}$ equal to zero.

Rudkevich proves that if the players begin by bidding their true costs and that if each player updates according to the strategy just described then, in the limit, the supply functions converge to the SFE under $R$-parametrization. That is, Rudkevich not only finds the equilibrium, but also provides an explanation of how bidders might converge to it.

There are three principal drawbacks to the approaches in [1], [2], [5]. The first is the assumption that the true linear cost coefficient $c_{k}$ is zero. This assumption can be relaxed and the analysis of Green and Newbery generalized to the case of non-symmetric players with non-zero values of $c_{k}$ that differ from player to player [14].

The second drawback is that, in several BBP markets other than the England and Wales pool, players can bid different supply functions for each half-hour or hour long pricing-period over a day and can bid piecewise constant or piecewise linear supply functions. That is, combining data from several pricing-periods under the assumption that the data all corresponds to the same underlying quadratic bid cost function is not likely to be a good model of realistic behavior, unless other constraints, such as regulatory oversight, limit the ability to bid different cost functions period by period. Finally, Green and Newbery and Rudkevich do not explain how to incorporate transmission constraints into their approaches.

Hobbs and his colleagues [6], [7] formulate the problem of calculating SFE in the presence of transmission constraints as a "mathematical program with equilibrium constraints" [17]. They use a single pricing-period model; that is, they do not assume that the supply function must be the same across multiple pricing-periods.

Hobbs et al. [7] observe that there are likely to be multiple SFE equilibria under $(R, c)$-parametrization and then proceed, for the transmission constrained case they consider, to calculate the (apparently) unique SFE under $R$ parametrization and the (apparently) unique SFE under $c$ parametrization [7, Section 2]. However, as argued above, the restriction to $R$ - or $c$-parametrization is artificial, so even when there is a unique $\mathrm{SFE}$ under $c$-parametrization, say, this does not imply that the unique SFE under $c$ parametrization will actually be observed as an outcome of player behavior.

Younes and Ilic [3] consider an $(R \propto c)$-parametrization model with constant demand and solve for the SFE under $(R \propto c)$-parametrization. They also consider inter-temporal effects and transmission constraints.

Weber and Overbye [4] also consider an $(R \propto c)$ -

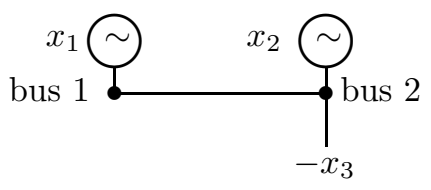

Fig. 1. Example system from [4] and described in section IV.

parametrization model and propose an iterative algorithm to find the SFE under $(R \propto c)$-parametrization. They imply that the choice of parametrization is not critical to the outcome by observing that "It can actually be shown that varying both slope and intercept will not result in increased personal welfare" $[4, \S 7]$. In particular, Weber and Overbye implicitly argue that, given fixed bid cost functions for everyone else, the particular parametrization of a player $k$ 's bid cost function is not critical.

In the rest of this paper, I will argue using Weber and Overbye's example that the parametrization is critical to the results, even in the transmission unconstrained case. The Weber and Overbye example is used because it is simple enough to be analyzed directly. Similar observations also apply to the results reported in [3], [6], [7].

I will show that, under the apparently more realistic $(R, c)$-parametrization, multiple Nash equilibria are almost inevitable if players can bid different bid cost functions in each pricing-period and there is no uncertainty. This applies to both a transmission unconstrained and a transmission constrained version of the model and illustrates, in the context of a BBP market, the observation made by Klemperer and Meyer in [8].

As Stoft points out in [18], the existence of multiple Nash equilibria is very problematic from the perspective of trying to predict behavior. In some cases, one of the equilibria may be preferred by everyone to all the other equilibria. Such an equilibrium is called a "focal equilibrium" $[19$, Section 6.3]. If the players realize the existence of the focal equilibrium then this equilibrium may actually prevail. We will consider the focal equilibrium under $(R, c)$-parametrization for the Weber and Overbye example. We will find that the focal equilibrium is equivalent to the Cournot equilibrium for this example system.

\section{TRANSMISSION UNCONSTRAINED EXAMPLE SYSTEM}

In this section, I develop the example system of Weber and Overbye [4]. There is a transmission unconstrained and a transmission constrained version. I will first consider the transmission unconstrained version. The transmission constrained version will be considered in section $\mathrm{V}$.

The example system is described in detail in section IVA. Then in sections IV-B through IV-F, respectively, I display solutions for: the competitive case (all players perfectly competitive, bidding $f_{k} \equiv \hat{f}_{k}, k=1,2,3$ ); SFE for $(R \propto c)$-parametrization; SFE for $c$-parametrization; SFE for $(R, c)$-parametrization; and Cournot equilibrium. The solutions are discussed in section IV-G.

\section{A. Description}

I quote the details of the example system of Weber and Overbye [4, Section 6.1], transcribing their notation. There 


\begin{tabular}{|c|c|c|c|c|c|c|c|c|}
\hline $\begin{array}{c}R_{1} \\
\frac{\$}{(\mathrm{MW})^{2} \mathrm{~h}}\end{array}$ & $\begin{array}{c}R_{2} \\
\frac{\$}{(\mathrm{MW})^{2} \mathrm{~h}}\end{array}$ & $\begin{array}{c}c_{1}^{\star} \\
\frac{\$}{M W h}\end{array}$ & $\begin{array}{c}c_{2}^{\star} \\
\frac{\$}{M W h}\end{array}$ & $\begin{array}{c}x_{1}^{\star} \\
\text { MW }\end{array}$ & $\begin{array}{c}x_{2}^{\star} \\
\text { MW }\end{array}$ & $\begin{array}{c}\lambda^{\star} \\
\$ \\
\mathrm{MWh}\end{array}$ & $\begin{array}{l}\pi_{1} \\
\$ \\
\mathrm{~h}\end{array}$ & $\begin{array}{l}\pi_{2} \\
\frac{\$}{h}\end{array}$ \\
\hline 0.02 & 0.02 & 11.63 & 11.63 & 102.0 & 102.0 & 13.67 & 270.7 & 270.7 \\
\hline 0.02 & 0.04 & 12.32 & 9.55 & 86.82 & 112.5 & 14.05 & 276.2 & 329.3 \\
\hline 0.04 & 0.04 & 10.65 & 10.65 & 96.77 & 96.77 & 14.51 & 343.4 & 343.4 \\
\hline 0.1 & 0.1 & 6.83 & 6.83 & 89.11 & 89.11 & 15.74 & 432.3 & 432.3 \\
\hline 1.0 & 0.1 & -83.91 & 9.59 & 100.4 & 68.76 & 16.47 & 548.6 & 397.5 \\
\hline 1.0 & 1.0 & -61.31 & -61.31 & 78.72 & 78.72 & 17.41 & 521.1 & 521.1 \\
\hline 10.0 & 10.0 & -753.5 & -753.5 & 77.11 & 77.11 & 17.66 & 531.4 & 531.4 \\
\hline 100.0 & 100.0 & -767.7 & -767.7 & 76.94 & 76.94 & 17.69 & 532.4 & 532.4 \\
\hline
\end{tabular}

TABLE I

SFE VALUES OF STRATEGIC

VARIABLES FOR

c-PARAMETRIZATION AND

CORRESPONDING VALUES OF

GENERATIONS, PRICES, AND

PROFITS, FOR VARIOUS CHOICES OF

THE QUADRATIC COEFFICIENTS FOR

EXAMPLE IN SECTION IV-D.

are two strategic players, at buses 1 and 2, respectively. Let $x_{k}$ be the generation by player $k$ at bus $k$. In addition, there is a demand at bus 2 that is assumed to bid honestly. That is, it is competitive. I will denote its variable by $x_{3}$. The situation is depicted in figure 1.

The true cost (and negative benefit) functions are $\hat{f}_{k}$ : $\mathbb{R} \rightarrow \mathbb{R}, k=1,2,3$, defined by:

$$
\begin{aligned}
& \forall x_{1}, \hat{f}_{1}\left(x_{1}\right)=\frac{1}{2} 0.02\left(x_{1}\right)^{2}+10 x_{1}, \\
& \forall x_{2}, \hat{f}_{2}\left(x_{2}\right)=\frac{1}{2} 0.02\left(x_{2}\right)^{2}+10 x_{2}, \\
& \forall x_{3}, \hat{f}_{3}\left(x_{3}\right)=\frac{1}{2} 0.08\left(x_{3}\right)^{2}+30 x_{3} .
\end{aligned}
$$

I assume that these costs are denominated in $\$ / \mathrm{MWh}$. The assumption that the demand is competitive means that $f_{3} \equiv \hat{f}_{3}$. Initially, the network has no inequality constraints so that the only constraint is the energy balance constraint:

$$
x_{1}+x_{2}+x_{3}=0
$$

The pool solves the economic dispatch problem and the marginal price is determined. I will denote the price paid to the players as $\lambda$. I denote the profits to the players as $\pi_{1}$ and $\pi_{2}$, respectively.

\section{B. Competitive case}

If all players behave competitively by bidding their true costs then the optimal dispatch solution is $x_{1}^{\star}=x_{2}^{\star}=$ 111.1 MW, with a price of $\lambda^{\star}=12.22 \$ / \mathrm{MWh}$, and profit to player 1 and to player 2 is $\pi_{1}=\pi_{2}=123.5 \$ / \mathrm{h}$.

\section{SFE for $(R \propto c)$-parametrization}

Weber and Overbye formulate an $(R \propto c)$-parametrization model by requiring $R_{k}$ and $c_{k}$ to be related in the same way as the true parameters $\hat{R}_{k}=0.02$ and $\hat{c}_{k}=10$ are related for $k=1,2$. That is, they allow players 1 and 2 to multiply their true cost function by an arbitrary non-negative constant. Weber and Overbye present an iterative algorithm to solve for the SFE and report an SFE under $(R \propto c)$ parametrization for the values:

$$
R_{k}^{\star}=1.1502 \hat{R}_{k}, c_{k}^{\star}=1.1502 \hat{c}_{k}, k=1,2 .
$$

Corresponding to these values are generations of $x_{1}^{\star}=x_{2}^{\star}=$ 101.1 MW, price of $\lambda^{\star}=13.83 \$ / \mathrm{MWh}$, and profit to player 1 and to player 2 of $\pi_{1}=\pi_{2}=284.7 \$ / \mathrm{h}$. The profit is considerably higher than in the competitive case.

\section{SFE for c-parametrization}

In this section I will derive the conditions for SFE under $c$-parametrization, given fixed bid values of $R_{k}$, and then exhibit the corresponding equilibrium values of $c_{k}, x_{k}, \lambda$, and $\pi_{k}$.

Because the only constraint in the example is the energy balance constraint, the demand variable can be eliminated in terms of $x_{1}$ and $x_{2}$. The pool problem is convex and its solution is given by the solution of the first order KarushKuhn-Tucker conditions, which yield:

$$
\begin{aligned}
{\left[\begin{array}{l}
x_{1} \\
x_{2}
\end{array}\right]=} & \frac{1}{R_{1} R_{2}+0.08\left(R_{1}+R_{2}\right)} \times \\
& {\left[\begin{array}{cc}
R_{2}+0.08 & -0.08 \\
-0.08 & R_{1}+0.08
\end{array}\right]\left[\begin{array}{c}
30-c_{1} \\
30-c_{2}
\end{array}\right], } \\
\lambda= & R_{1} x_{1}+c_{1}=R_{2} x_{2}+c_{2} .
\end{aligned}
$$

Given fixed choices for $R_{1}, R_{2}$, the profit function for player 1 is a concave function of $c_{1}$. Differentiating this function with respect to $c_{1}$ and setting equal to zero yields a linear equation in $c_{1}$ and $c_{2}$ (for fixed $R_{1}$ and $R_{2}$ ). Similarly for player 2. Any simultaneous solution of these linear equations satisfies the first order conditions for both profit maximization problems for the given values of $R_{1}$ and $R_{2}$. Since the objectives of both problems are concave and differentiable, any solution of the linear equations is an SFE.

In table I, the SFE values of the strategic variables and the corresponding values of generations, prices, and profits, are tabulated for various choices of the quadratic coefficients $R_{1}$ and $R_{2}$ that are greater than or equal to the true values of $\hat{R}_{1}=\hat{R}_{2}=0.02$.

For the larger values of $R_{1}$ and $R_{2}$, the SFE values of $c_{1}^{\star}$ and $c_{2}^{\star}$ are negative. It might be argued that these values would not be allowed in a pool since the bid cost function then has a negative derivative at zero power production. However, since pools typically allow piecewise linear or piecewise quadratic bid cost functions, a composite bid function could easily be created such that the bid cost function had non-negative derivative at zero production and was also convex. The corresponding composite supply function would have a "hockey stick" shape.

Figures 2 and 3 show the profits of, respectively, player 1 and player 2 at the SFE under $c$-parametrization versus $R_{1}$ and $R_{2}$, both in the range 0.0004 to 2 . Qualitatively, for any fixed $R_{2}$, the corresponding SFE profits $\pi_{1}$ for player 1 


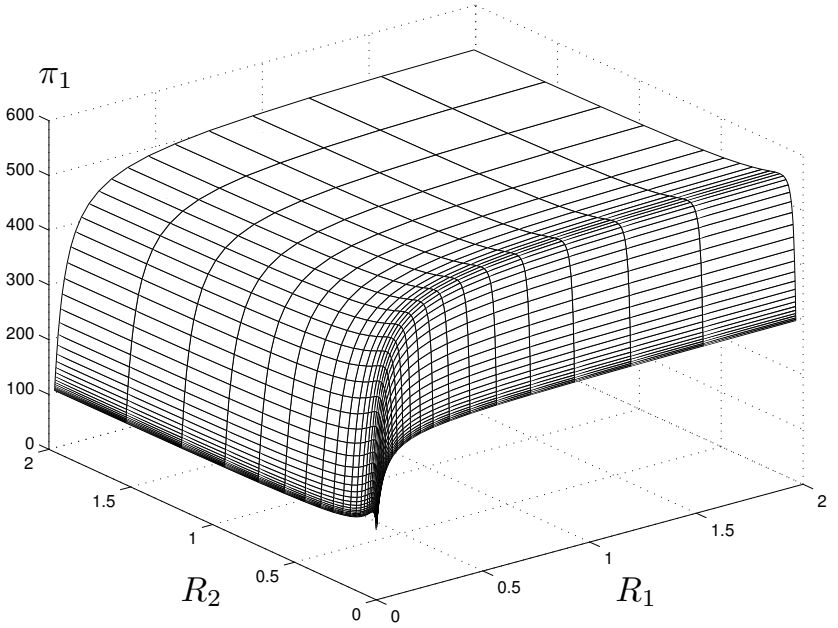

Fig. 2. SFE profit $\pi_{1}$ of player 1 versus values of $R_{1}$ and $R_{2}$ for example in section IV-A.

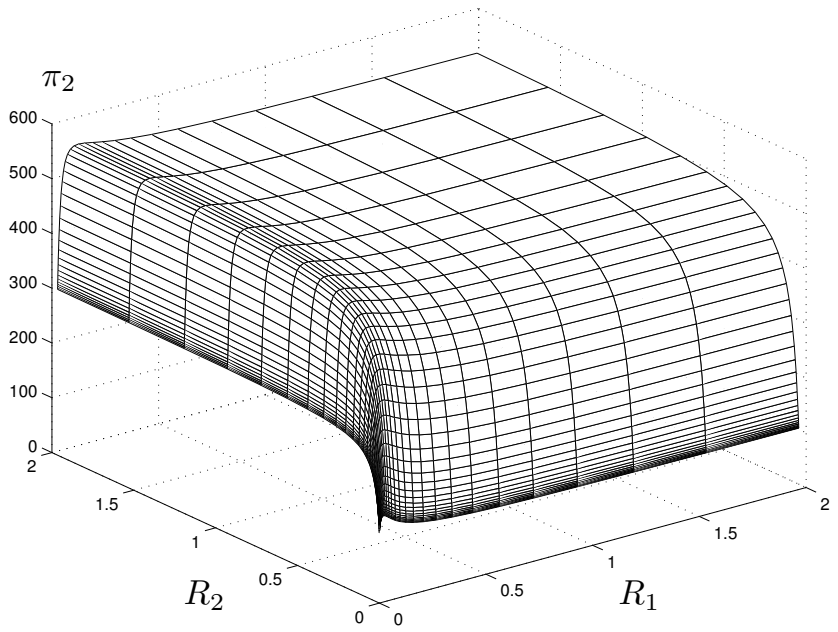

Fig. 3. SFE profit $\pi_{2}$ of player 2 versus values of $R_{1}$ and $R_{2}$ for example in section IV-A.

under $c$-parametrization are increasing in $R_{1}$. Similarly for the profits $\pi_{2}$ of player 2 and $R_{2}$.

The increase in profits with $R_{1}$ for player 1 is due to the interaction between the choices of strategic variables for players 1 and 2. This is the reason why the Weber and Overbye observation about the choice of parametrization is misleading. If player 2 is assumed to hold its strategic variable $c_{2}$ constant, then the optimal profits for player 1 are independent of $R_{1}$; however, the value of $c_{2}$ and the resulting choice of $c_{1}$ by player 1 is no longer an SFE. A similar observation holds for the profits of player 2 .

For this example, at least, if the players are required to commit a priori to values of $R_{1}$ and $R_{2}$, then it is in each of their interests for them to choose values of $R_{1}$ and $R_{2}$ as large as possible, or at least in the "plateau" region where $R_{1}, R_{2} \geq 1$. The profits for each player tend to $\pi_{1}=\pi_{2}=532.5 \$ / \mathrm{MWh}$ as the values of $R_{1}$ and $R_{2}$ tend to infinity. As we will see, these profits are the same as the Cournot level of profits.
Interestingly, if player 2 chooses a value of $R_{2}$ around 0.1 then the SFE for high values of $R_{1}$ yields profits for player 1 that are above the Cournot profits. In this case, the profits for player 2 are well below the Cournot profits. This highlights the dependence of the results of this parametrization on the assumed values of $R_{k}$.

\section{E. SFE for $(R, c)$-parametrization}

It is straightforward to prove that each of the entries in table I can also be interpreted as an SFE under $(R, c)$-parametrization. Moreover, each corresponding pair of profits for players 1 and 2 as shown in figures 2 and 3, respectively, is achievable as an SFE under $(R, c)$ parametrization. Although each entry is an equilibrium under $(R, c)$-parametrization, it is clear that both players would prefer to be at an equilibrium where values of $R_{1}$ and $R_{2}$ were large. We will discuss the implications of this observation further in relation to a focal equilibrium.

\section{F. Cournot equilibrium}

In this case, the price for energy is:

$$
\lambda=30-0.08\left(x_{1}+x_{2}\right) .
$$

Solving for the condition of mutual profit maximization, the Cournot solution is $x_{1}^{\star}=x_{2}^{\star}=76.92 \mathrm{MW}$, with price $\lambda^{\star}=17.69 \$ / \mathrm{MWh}$, and profit to player 1 and to player 2 of $\pi_{1}=\pi_{2}=532.5 \$ / \mathrm{MWh}$.

\section{G. Discussion}

The profits in the Cournot equilibrium are the same as the limiting case of the SFE equilibrium for large $R_{1}$ and $R_{2}$. This is not surprising since as $R_{1}$ and $R_{2}$ become large, the bid cost functions of these players becomes less elastic. Such a limiting SFE is a focal equilibrium under $(R, c)$-parametrization because it is mutually beneficial to all the strategic players for them all to bid cost functions with large positive values for the quadratic parameters.

For this example, single pricing-period SFE analysis that either assumes or predicts values of $R_{1}$ or $R_{2}$ that are less than one is completely unrealistic in that it seriously underestimates, by a factor of nearly two, the profits achievable to the players when they pick larger values of $R_{1}$ and $R_{2}$. The results for SFE reported in the literature for $c^{-}, R-$, and $(R \propto c)$-parametrization are potentially artifacts of the assumed parametrization and not indicative of the SFE under the more realistic $(R, c)$-parametrization. The results show that the profits vary from competitive to above Cournot profits depending on the assumed values of fixed parameters in the models.

I hypothesize that both the Cournot equilibrium and the SFE under $c$-parametrization (for large values of $R)$ are in general close to the focal SFE under $(R, c)$ parametrization. On the other hand, the SFEs under $(R \propto c)$-parametrization do not predict the focal equilibrium.

These observations for the single pricing-period model should be contrasted with the multiple pricing-period models. In a multiple pricing-period model where each player 
must bid a quadratic cost function that applies over several pricing-periods, then it can be the case that under $(R, c)$-parametrization it is optimal for a bidder $k$ to reveal the value of $c_{k}$ honestly [5], [14]. In this case, an $R$ parametrization model can be used to calculate the SFE.

\section{TRANSMission CONSTRAINED EXAMPLE SYSTEM}

In this section, I will consider the transmission constrained version of the Weber and Overbye example considered in the previous section. In [4], Weber and Overbye impose an $80 \mathrm{MW}$ transmission constraint on the line joining generator 1 to the demand. Generator 2 is located at the demand, so the transmission constraint limits production from generator 1 to no more than $80 \mathrm{MW}$. (An alternative interpretation is that generator 1 has an upper production limit of $80 \mathrm{MW}$ that has been honestly declared to the pool and is represented in the pool problem.)

\section{A. SFE for $(R \propto c)$-parametrization}

Weber and Overbye show that there is no pure strategy $\mathrm{SFE}$ under $(R \propto c)$-parametrization in this transmission constrained case. They observe that the profit function for one of the players is not concave in its decision variable. They exhibit a mixed strategy equilibrium.

\section{B. SFE for c-parametrization}

Because of the transmission constraint, the derivatives of the profit functions for each player are no longer linear functions of the strategic variables as in section IV-D but are instead piece-wise linear. However, it turns out that for this example the profit function for each player is still concave in the player decision variable. This contrasts with the case for $(R \propto c)$-parametrization where the profit function is not concave.

The concavity of profits and piecewise linearity of the derivatives of the profits under $c$-parametrization makes it relatively simple to find equilibria for this example, if they exist. The two possible cases are that either:

1. an SFE occurs for values of the decision variables that make the transmission constraint not binding, or

2. an SFE involves a binding transmission constraint.

Based on this observation, the SFE can be sought for various values of $R_{1}$ and $R_{2}$. In fact, there is a pure strategy SFE for all positive values of $R_{1}$ and $R_{2}$. The results are shown in figures 4 and 5 . These figures differ from figures 2 and 3 only in the region where $R_{2}$ is small enough such that the corresponding unconstrained SFE would result in a flow on the transmission line of greater than $80 \mathrm{MW}$.

The main qualitative difference is that the plateau region for player 2 occurs for any value of $R_{2}$ so long as player 1 chooses a value of $R_{1}$ that is above about 0.5 . Binding transmission constraints can support high equilibrium profits even at more modest levels of $R_{1}$ and $R_{2}$.

In general, when there are binding transmission constraints in the system, marginal cost prices may differ across busses. However, the optimal choice of decision variable by player 1 is such that the price is uniform in the sys-

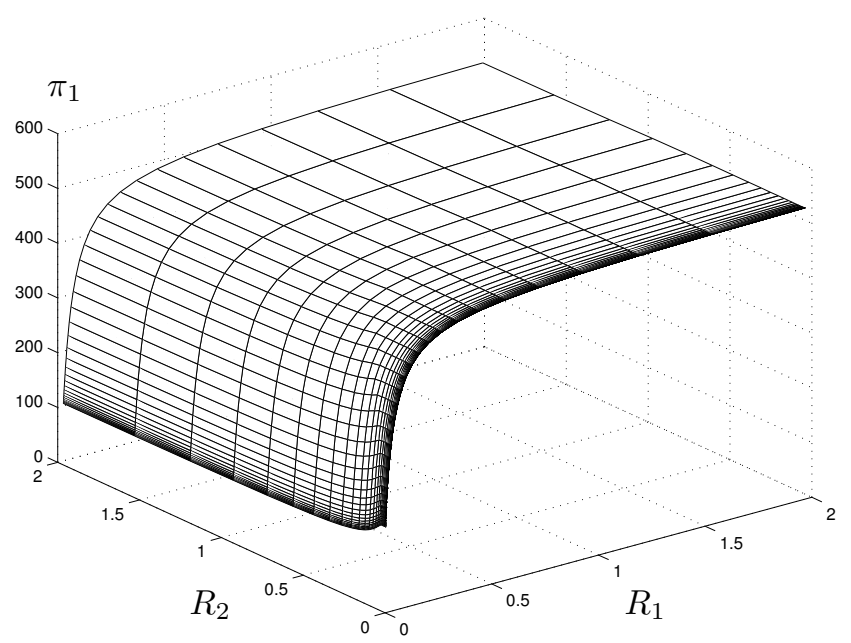

Fig. 4. SFE profit $\pi_{1}$ of player 1 versus values of $R_{1}$ and $R_{2}$ for transmission constrained example in section V.

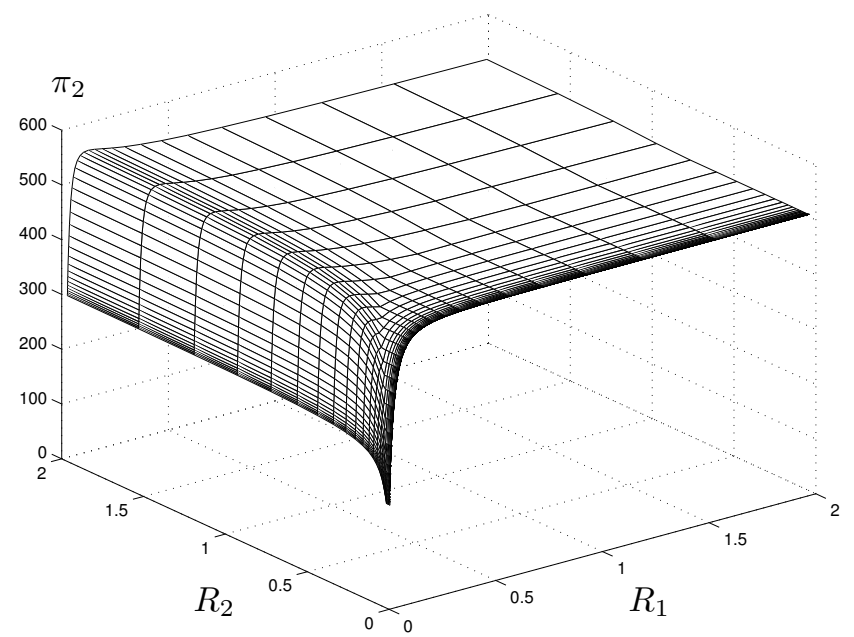

Fig. 5. SFE profit $\pi_{2}$ of player 2 versus values of $R_{1}$ and $R_{2}$ for transmission constrained example in section $\mathrm{V}$.

tem. This means that player 1 is capturing the congestion rental of the line.

\section{SFE for $(R, c)$-parametrization}

As previously, every point in figures 4 and 5 can be interpreted as a pure strategy $\mathrm{SFE}$ under $(R, c)$ parametrization.

\section{Cournot equilibrium}

The transmission unconstrained Cournot solution yields a flow on the line of less than $80 \mathrm{MW}$. This solution is also the Cournot equilibrium in the case of the $80 \mathrm{MW}$ transmission constraint.

\section{E. Discussion}

While there is no pure strategy SFE under $(R \propto c)$ parametrization, there are pure strategy SFEs under $c$ parametrization (and consequently pure strategy SFEs un- 
der $(R, c)$-parametrization, $)$ and there is a pure strategy Cournot equilibrium such that the transmission constraint is not binding. As $R_{1}$ and $R_{2}$ approach infinity, the profits again approach the Cournot profits. Since the players mutually benefit from large values of $R_{1}$ and $R_{2}$, it is reasonable that they could reach an equilibrium that does not load the line to its capacity. Again, the focal SFE under $(R, c)$-parametrization is equivalent to the Cournot equilibrium and to the SFE under $c$-parametrization for large values of $R$.

This example demonstrates that even the existence or non-existence of pure strategy equilibria can be an artifact of the choice of parametrization. In this case, $(R \propto$ $c)$-parametrization predicts no pure strategy equilibrium, whereas the focal SFE under $(R, c)$-parametrization is a pure strategy equilibrium.

\section{Conclusion}

I have demonstrated with examples that some SFE results presented in the literature are in fact artifacts of assumptions about the choices of particular bid parameters. At least in the examples considered, unfettered choices of convex quadratic bid cost functions can be expected to lead to results that are closer to Cournot outcomes, although the resulting bid cost functions can sometimes have very large values for their quadratic coefficients and negative values for their linear coefficients. I hypothesize that this is true in more general cases.

Nevertheless, exercise of market power on a period by period basis potentially requires significantly different bid cost functions for each period. Regulatory oversight may discourage bidding of such widely varying cost functions or discourage bid costs with large values of quadratic coefficients or explicitly cap the maximum price for energy that can be charged. Market rules could be designed to explicitly restrict the bidding flexibility by, for example:

- limiting the maximum values of the quadratic coefficients in the bid cost functions, or

- requiring bids to be consistent across multiple pricingperiods.

Both of these requirements would significantly reduce the equilibrium prices. The second point has been highlighted by Green and Newbery $[1, \S I I . B]$ and Baldick and Hogan [15]. Reasonable upper bounds on the allowed values of quadratic coefficients could be determined from historic operating data. Moreover, uncertainty in demand or in the the operational status of other players can reduce the desirability of bidding cost functions with large quadratic coefficients because of the risk of not being dispatched [20].

Another issue is that strategic generators were able to capture all the congestion rental of the constrained line. As discussed by Oren [12] and Stoft [18], this may be an artifact of the lack of modeling of a market for transmission congestion contracts.

\section{ACKNOWLEDGMENT}

This work was funded in part by the National Science Foundation under grant grant ECS-0082506. The author would like to thank Steven Stoft for suggesting some of the ideas in this paper and would also to thank Alex Rudkevich for comments and suggestions.

\section{REFERENCES}

[1] Richard Green and David M. Newbery, "Competition in the British electricity spot market," Journal of Political Economy, vol. 100, no. 5, pp. 929-953, October 1992.

[2] Richard Green, "Increasing competition in the British electricity spot market," The Journal of Industrial Economics, vol. XLIV, no. 2, pp. 205-216, June 1996.

[3] Ziad Younes and Marija Ilic, "Generation strategies for gaming transmission constraints: Will the deregulated electric power market be an oligopoly," in the Hawaii International Conference on System Sciences, Kona, Hawaii, January 6-9, 1998.

[4] J. D. Weber and T. J. Overbye, "A two-level optimization problem for analysis of market bidding strategies," in Proceedings of the IEEE Power Engineering Society Summer Meeting, Edmonton, Alberta, July 1999.

[5] Aleksandr Rudkevich, "Supply function equilibrium in poolco type power markets: Learning all the way," Tech. Rep. TCA Technical Paper Number 1299-1702, Tabors Caramanis and Associates, December 1999.

[6] Carolyn A. Berry, Benjamin Hobbs, William A. Meroney, Richard P. O'Neill, and William R. Stewart, Jr., "Analyzing strategic bidding behavior in transmission networks," in IEEE Tutorial on Game Theory Applications in Power Systems, H. Singh, Ed., pp. 7-32. IEEE, 1999.

[7] Benjamin Hobbs, Carolyn Metzler, and Jong-Shi Pang, "Strategic gaming analysis for electric power networks: An MPEC approach," IEEE Transactions on Power Systems, vol. 15, no. 2, pp. 638-645, May 2000.

[8] Paul D. Klemperer and Margaret A. Meyer, "Supply function equilibria in oligopoly under uncertainty," Econometrica, vol. 57, no. 6, pp. 1243-1277, November 1989.

[9] Judith B. Cardell, Carrie Cullen Hitt, and William W. Hogan, "Market power and strategic interaction in electricity networks," Resource and Energy Economics, vol. 19, no. 1-2, pp. 109-137, March 1997.

[10] William W. Hogan, "A market power model with strategic interaction in electricity markets," The Energy Journal, vol. 18, no. 4, pp. 107-141, 1997.

[11] William W. Hogan, Steven Stoft, and Shmuel S. Oren, "A transmission pricing debate," The Electricity Journal, vol. 10, no. 8, pp. 86-99, October 1997.

[12] Shmuel S. Oren, "Economic inefficiency of passive transmission rights in congested systems with competitive transmission," The Energy Journal, vol. 18, no. 1, pp. 63-83, 1997.

[13] Edward P. Kahn, "Numerical techniques for analyzing market power in electricity," The Electricity Journal, pp. 34-43, July 1998.

[14] Ross Baldick, Ryan Grant, and Edward P. Kahn, "Linear supply function equilibrium: Generalizations, application, and limitations," University of California Energy Institute POWER Paper PWP-078, www.ucei.berkeley.edu/ucei/PDF/pwp078.pdf, August 2000.

[15] Ross Baldick and William Hogan, "Capacity constrained supply function equilibrium models of electricity markets: Stability, non-decreasing constraints, and function space iterations," University of California Energy Institute POWER Paper PWP-089, www.ucei.berkeley.edu/ucei/PDF/pwp089.pdf, December 2001.

[16] Nils-Henrik Morch von der Fehr and David Harbord, "Spot market competition in the UK electricity industry," The Economic Journal, vol. 103, no. 418, pp. 531-546, May 1993.

[17] Zhi-Quan Luo, Jong-Shi Pang, and Daniel Ralph, Mathematical Programs with Equilibrium Constraints, Cambridge University Press, Cambridge, England, 1996.

[18] Steven Stoft, "How financial transmission rights curb market power," Manuscript, University of California Energy Institute, May 1997.

[19] Jean Tirole, The Theory of Industrial Organization, The MIT Press, Cambridge, Massachusetts and London, England, 1988.

[20] Mark O'Malley, Kado Miyakawa, Shane Rourke, and ChenChing Liu, "Strategic bidding in an optimal power flow electricity market," Submitted to IEEE Transactions on Power Systems, 2000. 